\title{
Vector meson production in coherent hadronic interactions: Update on predictions for energies available at the BNL Relativistic Heavy Ion Collider and the CERN Large Hadron Collider
}

\author{
V. P. Gonçalves ${ }^{1}$ and M. V. T. Machado ${ }^{2}$ \\ ${ }^{1}$ Instituto de Física e Matemática, Universidade Federal de Pelotas Caixa Postal 354, CEP 96010-900, Pelotas, RS, Brazil \\ ${ }^{2}$ Instituto de Física, Universidade Federal do Rio Grande do Sul Caixa Postal 15051, CEP 91501-970, Porto Alegre, RS, Brazil
}

(Received 15 June 2011; published 11 July 2011)

\begin{abstract}
In this Rapid Communication we update our predictions for the photoproduction of vector mesons in coherent $p p$ and $A A$ collisions at Relativistic Heavy Ion Collider (RHIC) and Large Hadron Collider (LHC) energies using the color dipole approach and the Color Glass Condensate formalism. In particular, we present our predictions for the first run of the LHC at half energy and for the rapidity dependence of the ratio between the $J / \Psi$ and $\rho$ cross sections at RHIC energies.
\end{abstract}

DOI: 10.1103/PhysRevC.84.011902

PACS number(s): 12.38.Bx, 13.60.Hb, 25.40.Ve, 25.75.Dw

The Large Hadron Collider (LHC) at CERN started 19 months ago. During this period a large amount of data have been collected considering $p p$ collisions at $\sqrt{s}=0.9,2.36$, and $7 \mathrm{TeV}$ as well as $P b P b$ collisions at $\sqrt{s}=2.76 \mathrm{TeV}$. Currently, there is a great expectation that LHC will discover the Higgs boson and whatever new physics beyond the Standard Model that may accompany it, such as supersymmetry or extra dimensions [1]. However, we should remember that LHC opens a new kinematical regime at high energy, where several questions related to the description of the high-energy regime of quantum chromodynamics (QCD) remain without satisfactory answers (for recent reviews see Ref. [2]). In recent years we have proposed the analysis of coherent collisions in hadronic interactions as an alternative way to study the QCD dynamics at high energies [3-11]. The basic idea in coherent hadronic collisions is that the total cross section for a given process can be factorized in terms of the equivalent flux of photons into the hadron projectile and the photon-photon or photon-target production cross section. The main advantage of using colliding hadrons and nuclear beams for studying photon-induced interactions is the high equivalent photon energies and luminosities that can be achieved at existing and future accelerators (for recent reviews see Ref. [12]). Consequently, studies of $\gamma p(A)$ interactions at the LHC could provide valuable information on the QCD dynamics at high energies.

Our goal in this Rapid Communication is to furnish an update of our predictions for the vector meson production in coherent $p p$ and $A A$ collisions, which can be used in the current and future analysis of the experimental data from RHIC and LHC. In particular, we will update Refs. [9,10], where we have studied the $J / \Psi, \Upsilon$, and $\rho$ production at RHIC and for the full energies of the LHC. Moreover, for the first time, we will present our predictions for the rapidity dependence of the ratio between the $J / \Psi$ and $\rho$ cross sections at RHIC energies, which is currently under analysis by the STAR collaboration. Before presenting our results, in what follows we will introduce a very brief explanation about the models used in our calculations and refer to Refs. [9,10] for more complete details.

Lets consider the hadron-hadron interaction at large impact parameter ( $b>2 R_{h}$, where $R_{h}$ is the hadron radius) and at ultrarelativistic energies. In this regime we expect the electromagnetic interaction to be dominant. In heavy ion colliders, the heavy nuclei give rise to strong electromagnetic fields due to the coherent action of all protons in the nucleus, which can interact with each other. In a similar way, it also occurs when considering ultrarelativistic protons in $p p(\bar{p})$ colliders. The cross section for the photoproduction of a vector meson $V$ in a coherent hadron-hadron collision is given by

$$
\sigma(h h \rightarrow V h)=2 \int_{\omega_{\min }}^{\infty} d \omega \int d t \frac{d N_{\gamma}(\omega)}{d \omega} \frac{d \sigma}{d t}\left(W_{\gamma h}, t\right),
$$

where $\frac{d N_{\gamma}(\omega)}{d \omega}$ is the equivalent photon flux, $\frac{d \sigma}{d t}$ is the differential cross section for the process $(\gamma h \rightarrow V h), \omega_{\min }=$ $M_{V}^{2} / 4 \gamma_{L} m_{p}, W_{\gamma h}^{2}=2 \omega \sqrt{S_{\mathrm{NN}}}$, and $\sqrt{S_{\mathrm{NN}}}$ is the c.m.s energy of the hadron-hadron system. Considering the requirement that photoproduction is not accompanied by hadronic interaction (ultraperipheral collision) an analytic approximation for the equivalent photon flux of a nuclei can be calculated, which is given by [12]

$$
\frac{d N_{\gamma}(\omega)}{d \omega}=\frac{2 Z^{2} \alpha_{\mathrm{em}}}{\pi \omega}\left[\bar{\eta} K_{0}(\bar{\eta}) K_{1}(\bar{\eta})+\frac{\bar{\eta}^{2}}{2} \mathcal{U}(\bar{\eta})\right],
$$

where $\omega$ is the photon energy, $\gamma_{L}$ is the Lorentz boost of a single beam, and $\eta=\omega b / \gamma_{L} ; K_{0}(\eta)$ and $K_{1}(\eta)$ are the modified Bessel functions. Moreover, $\bar{\eta}=\omega\left(2 . R_{h}\right) / \gamma_{L}$ and $\mathcal{U}(\bar{\eta})=K_{1}^{2}(\bar{\eta})-K_{0}^{2}(\bar{\eta})$. On the other hand, for proton-proton interactions, we assume that the photon spectrum is given by [13]

$$
\begin{aligned}
\frac{d N_{\gamma}(\omega)}{d \omega}= & \frac{\alpha_{\mathrm{em}}}{2 \pi \omega}\left[1+\left(1-\frac{2 \omega}{\sqrt{S_{N N}}}\right)^{2}\right] \\
& \times\left(\ln \Omega-\frac{11}{6}+\frac{3}{\Omega}-\frac{3}{2 \Omega^{2}}+\frac{1}{3 \Omega^{3}}\right),
\end{aligned}
$$

with the notation $\Omega=1+\left[\left(0.71 \mathrm{GeV}^{2}\right) / Q_{\min }^{2}\right]$ and $Q_{\min }^{2}=$ $\omega^{2} /\left[\gamma_{L}^{2}\left(1-2 \omega / \sqrt{S_{N N}}\right)\right] \approx\left(\omega / \gamma_{L}\right)^{2}$. The factor two in Eq. (1) takes into account the fact that the hadron can act as both target and photon emitter. The experimental separation for such events is relatively easy; as photon emission is coherent over the hadron and the photon is colorless, we expect the events to be characterized by intact recoiled hadron (tagged hadron) 
and a two rapidity gap pattern (for a detailed discussion see Ref. [12]).

We describe the vector meson production in the color dipole frame, in which most of the energy is carried by the hadron, while the photon has just enough energy to dissociate into a quark-antiquark pair before the scattering. In this representation the probing projectile fluctuates into a quark-antiquark pair (a dipole) with transverse separation $r$ long after the interaction, which then scatters off the hadron [14]. In the dipole picture the amplitude for production of a vector meson $V$ is given by (see, e.g., Refs. [14-16])

$$
\mathcal{A}_{T, L}^{\gamma^{*} h \rightarrow V h}\left(x, Q^{2}, \Delta\right)=\int d z d^{2} r\left(\Psi^{V *} \Psi\right)_{T, L} \mathcal{A}_{q \bar{q}}(x, \boldsymbol{r}, \Delta),
$$

where $\left(\Psi^{V *} \Psi\right)_{T, L}$ denotes the overlap of the photon and vector meson wave functions. The variable $z(1-z)$ is the longitudinal momentum fractions of the quark (antiquark), $\Delta$ denotes the transverse momentum lost by the outgoing hadron $\left(t=-\Delta^{2}\right)$, and $x$ is the Bjorken variable. Moreover, $\mathcal{A}_{q \bar{q}}$ is the elementary elastic amplitude for the scattering of a dipole of size $\boldsymbol{r}$ on the target. It is directly related to scattering amplitude $\mathcal{N}(x, \boldsymbol{r}, \boldsymbol{b})$ and consequently to the QCD dynamics (see below). One has that [16]

$$
\mathcal{A}_{q \bar{q}}(x, r, \Delta)=i \int d^{2} \boldsymbol{b} e^{-i \boldsymbol{b} . \Delta} 2 \mathcal{N}(x, \boldsymbol{r}, \boldsymbol{b}),
$$

where $\boldsymbol{b}$ is the transverse distance from the center of the target to one of the $q \bar{q}$ pair of the dipole. Consequently, one can express the amplitude for the exclusive production of a vector meson as follows:

$$
\begin{aligned}
\mathcal{A}_{T, L}^{\gamma^{*} h \rightarrow V h}\left(x, Q^{2}, \Delta\right)= & i \int d z d^{2} \boldsymbol{r} d^{2} \boldsymbol{b} e^{-i[\boldsymbol{b}-(1-z) \boldsymbol{r}] . \Delta} \\
& \times\left(\Psi_{V}^{*} \Psi\right)_{T} 2 \mathcal{N}(x, \boldsymbol{r}, \boldsymbol{b}),
\end{aligned}
$$

where the factor $[i(1-z) \boldsymbol{r}] . \boldsymbol{\Delta}$ in the exponential arises when one takes into account nonforward corrections to the wave functions [17]. Finally, the differential cross section for exclusive vector meson production is given by

$$
\frac{d \sigma_{T, L}}{d t}\left(\gamma^{*} h \rightarrow V h\right)=\frac{1}{16 \pi}\left|\mathcal{A}_{T, L}^{\gamma^{*} p \rightarrow V h}\left(x, Q^{2}, \Delta\right)\right|^{2}\left(1+\beta^{2}\right),
$$

where $\beta$ is the ratio of real to imaginary parts of the scattering amplitude. For the case of heavy mesons, skewness corrections are quite important, and they are also taken into account (for details, see Refs. [15,16]).

The scattering amplitude $\mathcal{N}(x, \boldsymbol{r}, \boldsymbol{b})$ contains all information about the target and the strong interaction physics. In the Color Glass Condensate formalism [18-20], it encodes all the information about the nonlinear and quantum effects in the hadron wave function. It can be obtained by solving an appropriate evolution equation in the rapidity $y \equiv \ln (1 / x)$. As in Refs. [9,10] we will assume that in the case of photon-nucleus interactions the scattering amplitude is given by $[21]$

$$
\mathcal{N}(x, \boldsymbol{r}, \boldsymbol{b})=\left\{1-\exp \left[-\frac{1}{2} A T_{A}(b) \sigma_{\mathrm{dip}}(x, r)\right]\right\},
$$

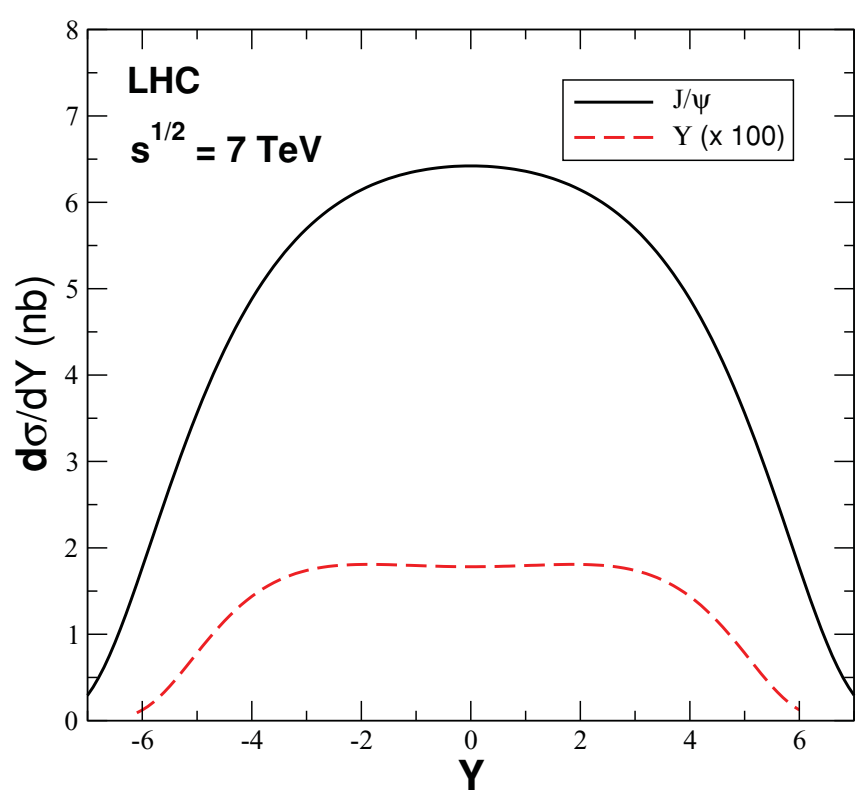

FIG. 1. (Color online) Predictions for the rapidity distribution of $J / \Psi$ and $\Upsilon$ photoproduction in $p p$ collisions at $\operatorname{LHC}(\sqrt{s}=7 \mathrm{TeV})$.

where $T_{A}(b)$ is the nuclear profile function (obtained from a three-parameter Fermi distribution for the nuclear density), and the dipole-nucleon cross section is taken from the Iancu, Itakura, and Munier (IIM) model [22].

In the case of photon-proton interactions we will use the nonforward saturation model of Ref. [23] (hereafter MPS model), which captures the main features of the dependence on energy, virtual photon virtuality, and momentum transfer $t$ and describes quite well the HERA data [24]. In the MPS model, the elementary elastic amplitude for dipole interaction is given by

$$
\mathcal{A}_{q \bar{q}}(x, r, \Delta)=\sigma_{0} e^{-B|t|} \mathcal{N}\left[r Q_{\text {sat }}(x,|t|), x\right],
$$

with the asymptotic behaviors $Q_{\text {sat }}^{2}(x, \Delta) \sim \max \left(Q_{0}^{2}, \Delta^{2}\right)$ $\exp [-\lambda \ln (x)]$. Specifically, the $t$ dependence of the saturation scale is parametrized as

$$
Q_{\text {sat }}^{2}(x,|t|)=Q_{0}^{2}(1+c|t|)\left(\frac{1}{x}\right)^{\lambda},
$$

in order to interpolate smoothly between the small and intermediate transfer regions. For the parameter $B$ we use the value $B=3.754 \mathrm{GeV}^{-2}$ [23]. Finally, the scaling function $\mathcal{N}$ is obtained from the forward saturation model [22].

Let us calculate the rapidity distribution and total cross section for the $J / \Psi$ and $\Upsilon$ photoproduction in coherent $p p$ collisions. The distribution on rapidity $Y$ of the produced final state can be directly computed from Eq. (1), by using its relation with the photon energy $\omega$, i.e., $Y \propto \ln \left(2 \omega / M_{V}\right)$. Explicitly, the rapidity distribution is written as

$$
\frac{d \sigma[h+h \rightarrow h \otimes V \otimes h]}{d Y}=\omega \frac{d N_{\gamma}(\omega)}{d \omega} \sigma_{\gamma h \rightarrow V h}(\omega),
$$

where $\otimes$ represents the presence of a rapidity gap. Consequently, given the photon flux, the rapidity distribution is thus 

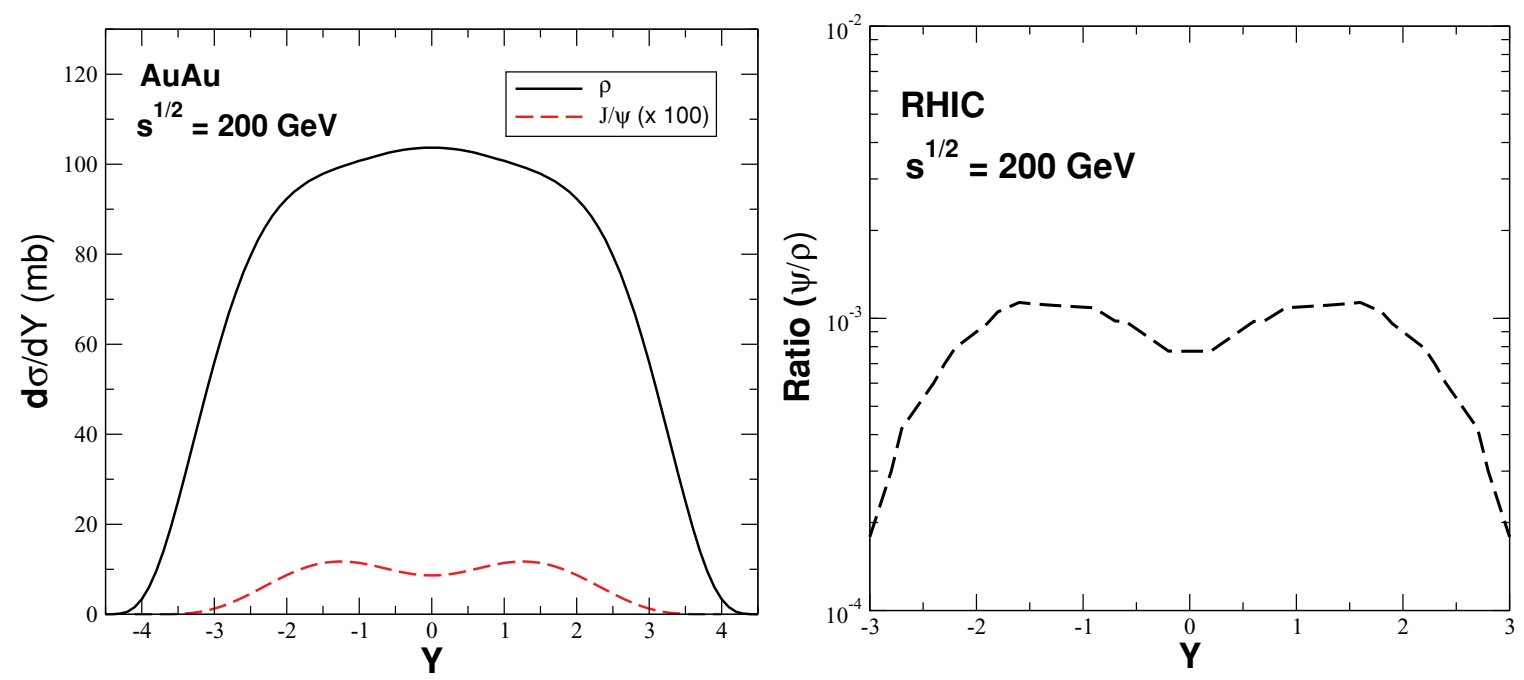

FIG. 2. (Color online) Predictions for the rapidity distribution of $\rho$ and $J / \Psi$ photoproduction in $A u A u$ collisions at RHIC (left panel) and for the dependence on the rapidity for the ratio between the $J / \Psi$ and $\rho$ cross sections (right panel).

a direct measure of the photoproduction cross section for a given energy.

In Fig. 1 we present our predictions for $p p$ collisions at $\sqrt{s}=7 \mathrm{TeV}$. The results are shown for the full rapidity range. The rapidity distribution at LHC probes a large interval of photon-proton center of mass energy since $W_{\gamma h}^{2} \simeq M_{V} \sqrt{s} \exp ( \pm Y)$, which corresponds to very small $x \simeq M_{V} e^{-Y} / \sqrt{s}$. Therefore, its experimental analysis can be useful to determine the QCD dynamics. As a reference, one has at central rapidity $\frac{d \sigma}{d y}(y=0) \simeq 6.5 \mathrm{nb}(18 \mathrm{pb})$ for $J / \Psi$ $(\Upsilon)$. In Table I we present our estimates for the integrated cross sections and production rates assuming the design luminosity $\mathcal{L}_{\mathrm{LHC}}^{\mathrm{pp}}=10^{7} \mathrm{mb}^{-1} \mathrm{~s}^{-1}$. In comparison with previous results presented in Ref. [9] our predictions at $\sqrt{s}=7 \mathrm{TeV}$ are almost a factor 2 (4) smaller than those obtained for $J / \Psi(\Upsilon)$ production at the full LHC energy. The difference between the factors for $J / \Psi$ and $\Upsilon$ production is directly associated with the distinct energy dependence predicted by the saturation physics, which implies larger effects in the $J / \Psi$ production.

In Fig. 2 and Table I we present our estimates for the $\rho$ and $J / \Psi$ production in ultraperipheral heavy ion collisions at RHIC. We assume $\mathcal{L}_{\text {RHIC }}=0.4 \mathrm{mb}^{-1} \mathrm{~s}^{-1}$. The results for $\rho$ production are consistent with those presented in Ref. [10]. In the case of $J / \Psi$ production, this is the first time that we present the predictions using the IIM model [22] as input in the calculations of the dipole-nucleus cross section. In comparison with the results presented in Ref. [5], where the

TABLE I. The integrated cross section (events rate/second) for vector meson photoproduction in $p p$ and $A A$ collisions at RHIC and LHC energies.

\begin{tabular}{lccc}
\hline \hline Meson & RHIC $(A u A u)$ & LHC $(P b P b)$ & LHC $(p p)$ \\
\hline$\rho$ & $609.7 \mathrm{mb}(256.0)$ & $4276 \mathrm{mb}(1796.0)$ & - \\
$J / \Psi$ & $0.51 \mathrm{mb}(0.20)$ & $20 \mathrm{mb}(8.40)$ & $63.70 \mathrm{nb}(637.0)$ \\
$\Upsilon$ & - & - & $0.18 \mathrm{nb}(1.80)$ \\
\hline \hline
\end{tabular}

GBW model [25] was used as input of the calculations, our predictions are $\approx 10 \%$ larger, which is directly associated with the difference between the description of the linear regime proposed by these two models. In the right panel of the Fig. 2 we present our prediction for the dependence on the rapidity for the ratio between the $J / \Psi$ and $\rho$ cross sections. This observable is currently under analysis by the STAR collaboration [26]. We are considering here the case without mutual nuclear excitation. The correction factor for meson production accompanied by mutual excitation is rather rapidity dependent and gives an overall suppression of $1 / 10$ in the integrated cross sections (almost the same for light and heavy mesons) [27]. In the case presented here, the ratio $J / \Psi / \rho$ should have small sensitivity to those corrections

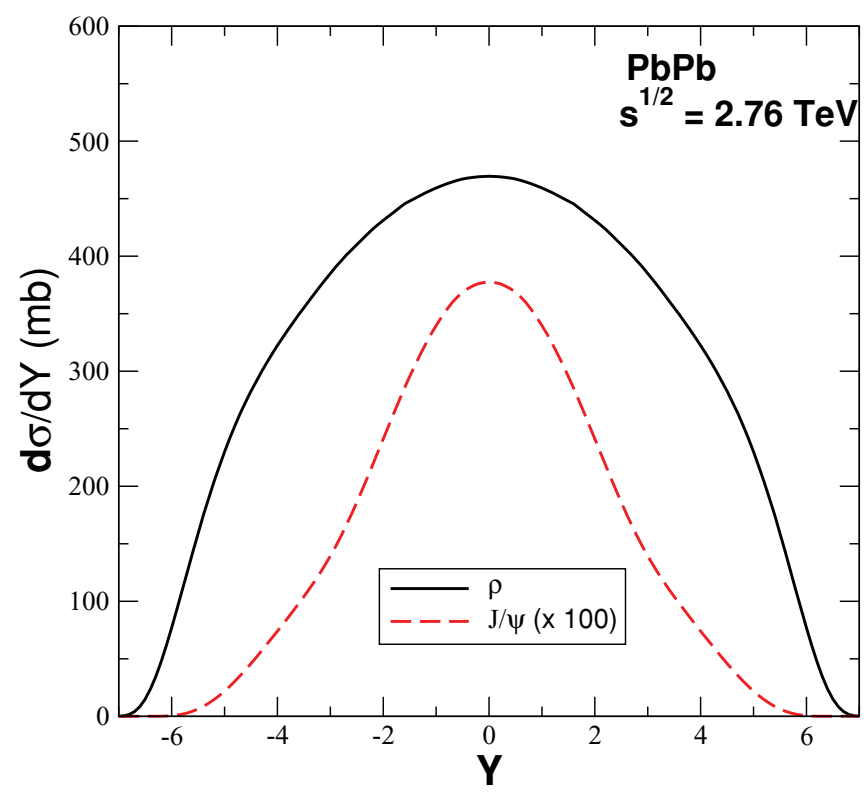

FIG. 3. (Color online) Predictions for the rapidity distribution of $\rho$ and $J / \Psi$ photoproduction in $P b P b$ collisions at LHC. 
and gives at central rapidity $\frac{d \sigma\left(\rho^{0}\right)}{d y} / \frac{d \sigma(J / \Psi)}{d y} \simeq 1.2 \times 10^{3}$. This is consistent with the ratio between the normalizations of the cross sections for vector meson production, which are proportional to $\left(M_{V}^{3} / m_{q}^{8}\right) \Gamma\left(V \rightarrow e^{+} e^{-}\right) W^{4 \lambda_{V}}$, where $\lambda_{V}$ is the effective energy power for each vector meson and $m_{q}$ is the relevant quark mass.

Finally, in Fig. 3 and Table I we present our predictions for the rapidity distribution of $\rho$ and $J / \Psi$ photoproduction in $\mathrm{PbPb}$ collisions in the first heavy ion run of the LHC $(\sqrt{s}=2.76 \mathrm{TeV})$. We assume the design luminosity $\mathcal{L}_{\mathrm{LHC}}^{\mathrm{PbPb}}=$ $0.42 \mathrm{mb}^{-1} \mathrm{~s}^{-1}$. In comparison with the previous results $[9,10]$, our predictions for $\rho$ production are a factor 1.4 smaller than those obtained for the full LHC energy. In the $J / \Psi$ case, our predictions are smaller by a factor about two. These differences are associated to the distinct contribution of the saturation effects for the $J / \Psi$ and $\rho$ production, which affects the energy dependence of the cross sections. As a reference, one has at central rapidity $\frac{d \sigma}{d y}(y=0) \simeq 470 \mathrm{mb}(3.8 \mathrm{mb})$ for meson $\rho(J / \Psi)$.

In summary, in this Rapid Communication we updated our predictions for the vector meson production in coherent interactions at RHIC and LHC. In particular, we furnished the predictions for the center-of-mass energies of the first runs of the LHC. Moreover, we present our predictions for the dependence on the rapidity for the ratio between the $J / \Psi$ and $\rho$ cross sections, which is currently under analysis. Our results demonstrate that the production rates are large at LHC, which implies the experimental study of this process is feasible.

This work was partially financed by the Brazilian funding agencies CNPq and FAPERGS. The authors thank Joakim Nystrand for useful comments and discussions.
[1] J. Ellis, Acta Phys. Pol. B 38, 1071 (2007).

[2] E. Iancu and R. Venugopalan, arXiv:hep-ph/0303204; H. Weigert, Prog. Part. Nucl. Phys. 55, 461 (2005); J. JalilianMarian and Y. V. Kovchegov, ibid. 56, 104 (2006).

[3] V. P. Goncalves and M. V. T. Machado, Eur. Phys. J. C 28, 71 (2003); 29, 37 (2003); V. P. Goncalves, M. V. T. Machado, and W. K. Sauter, ibid. 46, 219 (2006).

[4] V. P. Goncalves and M. V. T. Machado, Eur. Phys. J. C 31, 371 (2003).

[5] V. P. Goncalves and M. V. T. Machado, Eur. Phys. J. C 40, 519 (2005).

[6] V. P. Goncalves and M. V. T. Machado, Phys. Rev. D 71, 014025 (2005).

[7] V. P. Goncalves and M. V. T. Machado, Phys. Rev. C 73, 044902 (2006).

[8] V. P. Goncalves and M. V. T. Machado, Phys. Rev. D 75, 031502 (2007).

[9] V. P. Goncalves and M. V. T. Machado, Phys. Rev. D 77, 014037 (2008).

[10] V. P. Goncalves and M. V. T. Machado, Phys. Rev. C 80, 054901 (2009).

[11] V. P. Goncalves, M. V. T. Machado, and A. R. Meneses, Phys. Rev. D 80, 034021 (2009).

[12] G. Baur, K. Hencken, D. Trautmann, S. Sadovsky, and Y. Kharlov, Phys. Rep. 364, 359 (2002); C. A. Bertulani, S. R. Klein, and J. Nystrand, Annu. Rev. Nucl. Part. Sci. 55, 271 (2005); K. Hencken et al., Phys. Rep. 458, 1 (2008).

[13] M. Drees and D. Zeppenfeld, Phys. Rev. D 39, 2536 (1989).

[14] N. N. Nikolaev and B. G. Zakharov, Phys. Lett. B 332, 184 (1994); Z. Phys. C 64, 631 (1994).
[15] V. P. Goncalves and M. V. T. Machado, Eur. Phys. J. C 38, 319 (2004).

[16] H. Kowalski, L. Motyka, and G. Watt, Phys. Rev. D 74, 074016 (2006).

[17] J. Bartels, K. Golec-Biernat, and K. Peters, Acta Phys. Pol. B 34, 3051 (2003).

[18] E. Iancu, A. Leonidov, and L. McLerran, Nucl. Phys. A 692, 583 (2001); E. Ferreiro, E. Iancu, A. Leonidov, and L. McLerran, ibid. 701, 489 (2002).

[19] I. I. Balitsky, Nucl. Phys. B 463, 99 (1996); Y. V. Kovchegov, Phys. Rev. D 60, 034008 (1999).

[20] J. Jalilian-Marian, A. Kovner, L. McLerran, and H. Weigert, Phys. Rev. D 55, 5414 (1997); J. Jalilian-Marian, A. Kovner, A. Leonidov, and H. Weigert, ibid. 59, 014014 (1998); J. Jalilian-Marian, A. Kovner, and H. Weigert, ibid. 59, 014015 (1998); J. Jalilian-Marian, A. Kovner, A. Leonidov, and H. Weigert, ibid. 59, 034007 (1999); A. Kovner, J. G. Milhano, and H. Weigert, ibid. 62, 114005 (2000); H. Weigert, Nucl. Phys. A 703, 823 (2002).

[21] N. Armesto, Eur. Phys. J. C 26, 35 (2002).

[22] E. Iancu, K. Itakura, and S. Munier, Phys. Lett. B 590, 199 (2004).

[23] C. Marquet, R. B. Peschanski, and G. Soyez, Phys. Rev. D 76, 034011 (2007).

[24] V. P. Goncalves, M. V. T. Machado, and A. R. Meneses, Eur. Phys. J. C 68, 133 (2010).

[25] K. Golec-Biernat and M. Wüsthoff, Phys. Rev. D 59, 014017 (1998); 60, 114023 (1999).

[26] J. Seger, private communication.

[27] A. J. Baltz, S. R. Klein, and J. Nystrand, Phys. Rev. Lett. 89, 012301 (2002). 